\title{
INSTRUMENTATION FOR THE MUON BASED NEUTRINO SOURCE
}

\author{
J. Norem, C-X Wang ANL, Argonne, IL, 60439 \\ N. Solomey, IIT, Chicago IL 60616
}

\begin{abstract}
There are a number of novel instrumentation issues relating to the generation cooling and acceleration of high power muon beams. Specific issues are monitoring the cooling water in the magnet nearest the target to minimize corrosion, monitoring the behavior of the target after the impact of the beam, and following the beam through the cooling accelerator chain. The muon cooling system also produces a number of unique issues such as making beam profile measurements in the presence of large backgrounds, measuring these backgrounds, losses, and the angular momentum of the beam. We describe the techniques we intend to use, and some of the constraints involved in their implementation.
\end{abstract}

\section{INTRODUCTION}

The Study II design for a neutrino source considered instrumentation needs for the various components in order to determine if these devices wold constitute a constraint on the overall design[1]. We considered all components of the facility, determined the beam diagnostic needs and discussed possible solutions, then evaluated the possible costs of these solutions. This paper is a summary of this work, stressing new problems. A workshop was held on these problems[2]

Although the muon bunches are large and intense, the backgrounds are somewhat uncertain, and space is generally limited, there seemed to be a number of options for most systems which can produce useful useful results.

An active $R \& D$ program is underway to systematically look at useful options, however the Lab G facility at FNAL is just becoming operational at this time and new experimental data is somewhat limited.

This paper will consider each component of the neutrino source, describe the instrumentation issues and proposed solutions.

\section{PROTON DRIVER}

The proton driver for the Study II neutrino source is assumed to be the AGS, and no new instrumentation requirements were identified that would require significant perturbations on the extensive instrumentation of this machine.

\section{TARGET AND SOLENOID}

The mercury target operating in a $20 \mathrm{~T}$ solenoidal field is a significant extrapolation beyond present technology.
While the superconducting solenoid is fairly conventional, the target and inner solenoid present unique problems. The dynamics of the liquid metal target when hit by a high power beam pulse is just beginning to be understood, and some preliminary data is available from experiments.

The solenoid magnet is exposed the highest radiation level of any component exclusive of the target. Although the coil-in-conduit option is comparatively stable, a bitter magnet is more efficient, however it requires an electric potential where the cooling water meets the copper. This will require the cooling water to be monitored to insure the $\mathrm{pH}$ does not change enough to permit corrosion of the copper.

Experiments on the response of liquid targets to high power beams are underway at the AGS (E-951)[3]. These experiments use interferometric strain gauges and fast optical cameras. At high powers it seems that nonlinear effects occur in the target. A Schlieren / Ronchi strain gauge is being developed which can use a fast YAG laser to provide time resolution of a few ns[4].

\section{BUNCHING AND COOLING LINE}

The primary instrumentation problems occur in the bunching and cooling line. Since the amount of cooling produced in each individual cell is small, cell-to-cell measurements of cooling would require very high precision measurement of the emittance, with a required measuring error $\delta \varepsilon / \varepsilon$ t much less than $1 \%$ which is difficult.. We assume cooling will be monitored over many cells.

We assume that the primary problems with tune up will be understanding alignment and matching of the beam through the bunching and cooling sections. In addition to monitoring the centroid position (with profile monitors) and energy variations (with bunch timing), the Twiss parameters, or more easily, the variations of the Twiss parameters can also be measured with profile monitors. We assume that the primary tools will be profile monitors and pencil beams.

We have looked at the effects of loss of hydrogen or arc down of an rf cavity using analytical methods. These effects produce a mismatch immediately downstream of the malfunctioning component, but the subsequent development of the beam envelope is complicated by synchrotron motion as the envelope evolves. If instrumentation is located at a limited number of locations the changes in the beam envelope functions may be difficult to detect.

We do not anticipate that backgrounds would be a major problem. Backgrounds come primarily from three 
sources: the target, the rf cavities, and from the muons themselves. Since the muon beam is primarily a solenoid from the target all the way through to the accelerators, all particles entering the line with a perpendicular momentum less than $\sim 0.1 \mathrm{GeV} / \mathrm{c}$ will travel down the whole line. Most of these the secondary particles produced at the target will be pions, kaons and secondary protons, and since there is nothing limiting the momentum, even protons approaching the beam energy will be transported. In most cases it is straightforward to isolate the muons using timing and background subtraction.

A more troublesome background may come from dark currents and electrons produce in the rf cavities. The dark currents seem to be produced from field emission in the cavities, and, when they interact with the walls, copious $\mathrm{x}$ rays are produced.

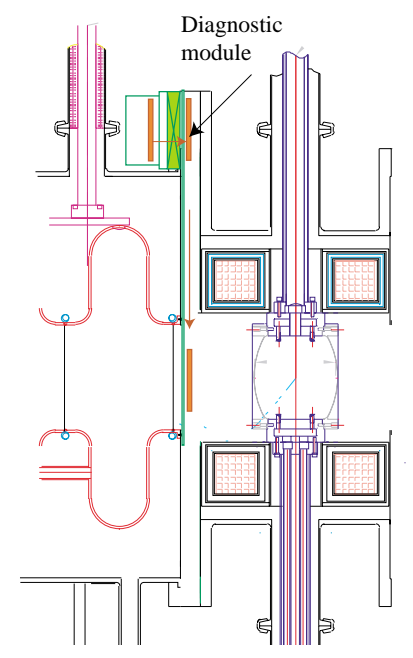

Figure 1, Inserting thin detectors in the cooling line.

It is desirable for rf and LH2 absorbers to occupy as much of the cell as possible, which leaves little room for instrumentation. The requirement that single modules can be removed from the line means that there must be expansion joints in the cooling line, and these can be used for diagnostic modules as shown in Figure 1. In principle whole or part of a complete cell could be used for instrumentation, however cost of magnet systems makes this somewhat undesirable.

Pencil beams will be very useful in tuning up all beam lines. At low energies collimators work very well to define 4 dimensional phase space, and reducing the voltage on the linac sections can reduce the longitudinal phase space to define a $6 \mathrm{D}$ pencil beam.

\subsection{Angular Momentum}

Angular momentum plays an important role in solenoid ionization cooling channels, unlike the quadrupole channels used everywhere else in high energy physics[5]. Due to the rotational symmetry of the solenoid focusing field, the canonical angular momentum is a conserved quantity when the cooling material is absent. When absorbers are added, however, the beam angular momentum can be changed and yield residual angular momentum at the end of a cooling channel. Non-zero beam angular momentum creates coupling between the two transverse degrees of freedom and causes problems for matching the beam into a following quadrupole channel. Furthermore, the angular momentum intrinsically couples with the beam emittance in the cooling dynamics. For efficient cooling in a periodic channel, it is necessary to keep the change in the net angular momentum to be zero. This requirement produces the condition, $\int \eta(s) \beta(s) B(s) d s=0$, where the integral is performed over one period, $B(\mathrm{~s})$ is the on-axis magnetic field, $\beta(\mathrm{s})$ is the envelope function, and $\eta(\mathrm{s})$ is the ionization energy loss rate.

Measurement of beam angular momentum is a new subject. A beam profile monitor, which measures beam distribution in $x$ and $y$, can measure the beam emittance but does not provide sufficient information to directly determine the angular momentum in a solenoid cooling channel. Based on beam emittance measurement, one may indirectly measure the angular momentum by identifying the two components that have different damping rates in the emittance damping curve. However, such measurements are difficult. Novel ideas are being explored to directly measure the correlation terms $\left\langle x p_{\mathrm{y}}\right\rangle$, etc.

The angular momentum will be measured using Cherenkov counters or slits to monitor the direction of the off axis orbits in the FODO lines downstream of the cooling line.

Since the energy loss rate, absorber thickness, and magnetic field are known, or can be measured with accuracy much better than one percent, and envelope function measurement is likely to be known to a few percent, it should be straightforward to determine whether the above requirement is approximately satisfied. Thus for machine operation purposes, beam angular momentum measurement is helpful but not absolutely necessary. For cooling demonstration experiments, however, good angular momentum measurement can provide further evidence for the cooling behavior. The ability to adjust the amount of material in absorbers is useful for correcting any residual angular momentum at the end of a cooling channel.

\subsection{Cooling Diagnostics}

Although the instrumentation for the cooling line in the neutrino source is somewhat straightforward, we also consider the additional requirement that these diagnostics systems should also work for a cooling experiment, where they must detect many fewer muons in an environment with x-rays and dark current electrons. We have been considering a number of options.

Faraday cups and secondary emission monitors have useful sensitivity and very fast time response and could be used in arrays to measure $x, y$ and $t$ profiles of bunches.

Silicon fibers are being considered both for single particle experiments and monitoring the complete beam. 
Bolometers located on the windows of the liquid hydrogen absorbers are being developed which can detect the $\mathrm{dE} / \mathrm{dx}$ energy deposition from the muon beam. This technique uses elements whose resistivity changes rapidly with temperature[6].

The possibility of $x-y$ arrays of detectors using thin plycrystalline CdTe semiconductor detectors has also been proposed by Placidi[7]. This technique should be capable of producing linear measurements of beam profile from one thin module.

\section{ACCELERATOR SYSTEMS}

Although the beams are larger than any used in existing accelerators, the problems encountered in tuning and operating the muon accelerator are similar to those seen at CEBAF and other laboratories. The main issues are: 1) operating the linac efficiently, to that all cavities are individually optimized, 2) optimizing the spreaders and combiners for minimum losses, 3) understanding the transverse optics of the return lines and linac focusing systems, (both linear and nonlinear) and 4) understanding the longidudinal development of the beam, (both linear and nonlinear) through the accelerator.

While most of the diagnostics can be done with standard profile, wall current and loss monitors, it is highly desirable to monitor the $6 \mathrm{D}$ development of the beam during acceleration and this may require the use of pencil beams.

\section{STORAGE RING}

The storage ring presents some new instrumentation problems. In addition to the usual emittance, divergence, closed orbit, injection, extraction, beam loss and beam energy measurements, it is necessary to measure the beam polarization and beam direction in the decay straight section as a function of time, to help determine the parameters of the secondary neutrino beam. The majority of instrumentation for the muon beam in the storage ring should utilize proven technology. The primary difficulties are caused by the required precision and the presence of decay electrons in the beam. We anticipate that the $6 \mathrm{D}$ "pencil" beams used to tune up the accelerator will also be useful in tuning up and operating the storage ring.

The fraction of primary decay electrons in the beam is given by $L / \gamma \tau c$, where $L$ is a path length in the storage ring, and $\gamma \tau \mathrm{c} \sim 126 \mathrm{~km}$, is the decay length at $20 \mathrm{GeV}$. In order to separate the contributions to the neutrino beam from decays in the upstream and downstream coupling sections, bending magnets have been introduced. Using a near detector, located a some distance from the straight section, the precise profile of the contributions from the coupling sections and decay straight can be evaluated with statistics $10^{5}$ larger than will be available in the far detector. We assume a dense, fine grained detector consisting of tungsten or other heavy plates interspersed with hodoscopes or liquid argon calorimeters. This could be located in a shaft, $100-200 \mathrm{~m}$ downstream of the decay straight, where the three "beams" would have a gaussian radius of about $1 \mathrm{~m}$. Rates could be high, on the order of 25 events/fill for a $1 \mathrm{~m}$ thick detector $(\sim 0.5 \mathrm{kHz})$.

The polarization of the muons in the storage ring can be measured by looking at the momentum distribution of the decay electrons moving in the beam direction. Roughly $8 \cdot 10^{4}$ decays/meter/turn will generate about $100 \mathrm{~W} / \mathrm{m}$ of signal. These decays produce a fan of electrons which are swept inward by the bending magnets. The muon beam polarization can be measured from the electron decay spectrum. A shower calorimeter, which can absorb the decay electrons of forward going electrons close to the beam, can be instrumented to look at the power deposition rate at the $\mu$ s timescale. Detection with a calorimeter should be relatively linear with electron energy. The precision with which the Monte Carlo calculations could be done would be crucial, and this work is underway.

\section{R\&D PROGRAM}

Since Lab G at Fermilab has become operational, we have begun an experimental program aimed at measuring the dark currents and $\mathrm{x}$ ray fluxes from the $805 \mathrm{MHz}$ cavity, and the response of all the proposed detector types in the magnetic, $x$ ray and soft electron environment.

\section{CONCLUSIONS}

We have done a preliminary survey of the instrumentation requirements of the neutrino source. Despite the unique nature of many of the problems, relatively conventional instrumentation, in particular profile monitors and techniques using pencil beams, could be applied in many places. Issues such as beam polarization in high intensity rings, precision measurements of the neutrino beams from the storage ring, measurement of angular momentum, and precise measurements of muons during cooling, studies of $\mathrm{rf}$ induced backgrounds and other problems will require further attention.

\section{ACKNOWLEDGEMENTS}

The workshop was supported by the Illinois Consortium on Accelerator Research with the support of T. Morrison of IIT. This work supported in part by the USDOE Office of High Energy Physics.

\section{REFERENCES}

[1] http://www.cap.bnl.gov/mumu/studyii/FS2report.html

[2] http://www.iit.edu/ bcps/hep/Nov2000 mucool.html

[3] http;//www.hep.princeton.edu/ mcdonald/mumu /target/targettrans29a.ps

[4] A. Hassanein and J. Norem. This conference

[5] K-J Kim and C-X Wang, PRL 85 (2000) 760.

[6] http://capp.iit.edu/ capp/workshops/muinst2000

/oreglia.pdf

[7] http://capp.iit.edu/ capp/workshops/muinst2000 /placidi.pdf 